\title{
A prática de exercícios físicos entre estudantes do curso de Medicina
}

\author{
The practice of physical exercises among Medical students \\ La práctica de ejercicios físicos en estudiantes de Medicina
}

\section{Resumo}

Introdução: A atividade física é essencial para uma vida saudável, com benefícios para a saúde física e mental. O sedentarismo pode ter consequências graves na vida do indivíduo, como o aumento da ansiedade e o agravamento da obesidade, hipertensão, e outras condições. O exercício físico também é fator de bem-estar psicológico, capaz de aliviar tensões e controlar sentimentos depressivos. No entanto, com o ingresso na universidade, principalmente no curso de Medicina, muitos estudantes possuem cada vez menos tempo destinado a essa prática. Objetivo: Identificar o perfil de prática de atividades físicas dos estudantes do curso de Medicina da Universidade Federal de Alfenas (UNIFAL-MG) em relação ao sexo e ao período cursado. Metodologia: Fizeram parte da pesquisa estudantes do curso de medicina que responderam a dois instrumentos de coleta de dados: I) Questionário Sociodemográfico e II) Questionário Internacional de Atividade Física - Forma Curta (IPAQ-SF). Esses questionários foram aplicados pelos pesquisadores no início e final do segundo e do terceiro períodos do curso, totalizando quatro inquéritos. Resultados: A análise de dados não demonstrou diferenças estatísticas das classificações entre homens e mulheres e entre cada período dentro do mesmo sexo. No entanto, observou-se uma diminuição da proporção de alunos no nível de atividade "ativo" em contraste com o aumento no nível "Irregularmente ativo A" aos finais dos períodos letivos. Conclusão: Ainda que considerando-se os desafios enfrentados durante a graduação, como acúmulo de atividades e exigências curriculares, não foi observada mudança do padrão de prática de exercícios físicos.

Palavras-chave: Graduação; Atividade física; Benefícios.

\section{Abstract}

Introduction: Physical activity is essential for a healthy life, with benefits for physical and mental health. Sedentary lifestyle can have serious consequences on the individual's life, such as increased anxiety and worsening obesity, hypertension, and other conditions. Physical exercise is also a factor of psychological well-being, capable of relieving 
tension and controlling depressive feelings. However, with the entry into the university, mainly in the medical course, many students have less and less time dedicated to this practice. Objective: To identify the profile of physical activity practice of medical students at the Federal University of Alfenas (UNIFAL-MG) in relation to gender and the period studied. Methodology: Medical students were included in the sample, who answered two data collection instruments: I) Sociodemographic Questionnaire and II) International Physical Activity Questionnaire - Short Form (IPAQ-SF). These questionnaires were applied by the researchers at the beginning and end of the second and third periods of the course, totaling four surveys. Results: Data analysis did not show statistical differences in gender classifications and between each period within the same sex. However, there was a decrease in the proportion of students in the level of activity "active" in contrast to the increase in the level "Irregularly active A" at the end of the school periods. Conclusion: Although considering the challenges faced during graduation, such as accumulation of activities and curricular requirements, there was no change in the pattern of physical exercise practice.

Keywords: Graduation; Physical activity; Benefits.

\section{Resumen}

Introducción: La actividad física es fundamental para una vida sana, con beneficios para la salud física y mental. El estilo de vida sedentario puede tener graves consecuencias en la vida del individuo, como un aumento de la ansiedad y el empeoramiento de la obesidad, la hipertensión y otras afecciones. El ejercicio físico es también un factor de bienestar psicológico, capaz de aliviar tensiones y controlar los sentimientos depresivos. Sin embargo, con el ingreso a la universidad, principalmente en la carrera de medicina, muchos estudiantes tienen cada vez menos tiempo dedicado a esta práctica. Objetivo: Identificar el perfil de la práctica de la actividad física de los estudiantes de medicina de la Universidad Federal de Alfenas (UNIFAL-MG) en relación con el género y el período estudiado. Metodología: Los estudiantes de Medicina fueron parte de la muestra, quienes respondieron a dos instrumentos de recolección de datos: I) Cuestionario Sociodemográfico y II) Cuestionario Internacional de Actividad Física - Forma Corta (IPAQ-SF). Estos cuestionarios fueron aplicados por los investigadores al inicio y al final del segundo y tercer período del curso, totalizando cuatro encuestas. Resultados: El análisis de los datos no mostró diferencias estadísticas en las clasificaciones de género y entre cada período dentro del mismo sexo. Sin embargo, hubo una disminución en la proporción de estudiantes en el nivel de actividad "activa" en contraste con el aumento en el nivel "Irregularmente activo A" al final de los períodos escolares. Conclusión: Aunque considerando los desafíos enfrentados durante la graduación, como la acumulación de actividades y los requisitos curriculares, no hubo cambio en el patrón de práctica del ejercicio físico.

Palabras clave: Graduación; Actividad física; Beneficios.

\section{Introdução}

A atividade física deve ser considerada uma parte essencial para um estilo de vida saudável, ao ponderar, principalmente, a sua relação com os benefícios gerados para a saúde física e mental (Reis, et al., 2000). Além disso, o exercício físico é uma forma de lazer e de restaurar a saúde dos efeitos nocivos que a rotina estressante do trabalho e do estudo traz.

A relação entre a prática de atividade física e a saúde teve início durante a era epidemiológica das doenças crônicodegenerativas. Durante esse período, por meio do paradigma da caixa-preta o sedentarismo foi associado de forma determinante com os agravos à saúde (Pitanga, 2008). Segundo Pate et al. (2008), o comportamento sedentário caracteriza-se pelas atividades que não causam aumento substancial do gasto de energia acima do nível de repouso e pode incluir atividades como dormir, sentar, deitar e assistir televisão, por exemplo.

A vida sedentária provoca, literalmente, o desuso dos sistemas funcionais. O sedentarismo é a principal causa do aumento da incidência de várias doenças, tais como hipertensão arterial, diabetes, obesidade, ansiedade, aumento do colesterol, infarto do miocárdio, dentre outras (Barros-Neto, 2003).

Além disso, uma correlação positiva também é encontrada entre bem-estar psicológico e a prática de exercícios físicos. Dessa forma, uma ruptura na sensação de bem-estar ou de satisfação com a vida pode estar associada ao manejo não adequado de eventos estressantes nas esferas pessoal, social ou cultural, afetando comportamentos e influenciando na percepção de saúde do indivíduo, o que pode trazer sofrimento pessoal (Silva et al., 2007).

A prática de atividades físicas tem sido amplamente utilizada como tratamento para doenças endócrinas, cardiovasculares e neurológicas. Porém, estudos recentes têm demonstrado que o treinamento físico também é seguro e 
adequado, tanto no curso, quanto após a terapia para um câncer primário (Alves et al., 2020). Outros estudos mostram também a influência positiva da atividade física sobre o sistema imunológico, assim como no quadro de pacientes com AIDS (Pitanga, 2008).

A prática de atividade física está associada ainda com o fenômeno da neuroplasticidade, o que resulta em novas conexões neurais, uma vez que, embora esse fenômeno seja mais comum na infância, o cérebro nunca cessa seu desenvolvimento. Assim, é importante a execução de atividades físicas, para melhor desenvolvimento cognitivo, devido aos estímulos sinápticos decorrentes de sua prática (Bonfim, et al., 2019).

Outro fator importante da prática regular de exercícios físicos está relacionado à qualidade e quantidade de sono, reduzindo o número de despertares ao longo de uma noite e diminuindo, ainda, a sonolência durante o dia. Além disso, as atividades físicas têm sido apontadas como um importante sincronizador do ritmo vigília-sono (Ropke, 2018).

O exercício físico tem se revelado eficaz tanto ao nível da saúde física quanto ao da mental, visto que, sua prática regular, além de contribuir para a redução de riscos cardiovasculares e de desenvolvimento de obesidade e diabetes, também ajuda no controle da ansiedade e dos sintomas depressivos. A atividade física tem sido considerada uma forma de aliviar emoções, tensões e frustrações adquiridas ao longo dos dias. Dessa maneira, o exercício físico regular pode melhorar a autoestima, o humor, a sensação de vigor e de bem-estar, além de propiciar uma maior capacidade de lidar com situações de tensão e estresse (Veigas \& Gonçalves, 2009).

Apesar da grande importância das atividades físicas sobre a qualidade de vida, existe uma tendência na redução de sua prática devido a diversos fatores, tais como o cotidiano atribulado da maioria das pessoas, o uso de tecnologias virtuais e as mudanças bruscas nas rotinas, como ocorre quando do ingresso em um curso de graduação.

Com o ingresso na universidade, muitos estudantes lidam pela primeira vez com a vida fora da casa dos pais ou familiares. Com isso, cabe a eles a organização dos estudos, cuidados com a moradia e atividades extracurriculares. Diante desse cenário, um dos desafios encontrados por esses estudantes está em conciliar a prática regular de atividades físicas com a rotina acadêmica.

Estudos realizados demonstraram um decréscimo na realização de atividades físicas ao longo dos anos na graduação, principalmente por acadêmicos do curso de medicina. Um dos estudos mostra uma diminuição nas atividades físicas vigorosas ao longo dos anos, com um consequente aumento nas atividades físicas moderadas, como as caminhadas (Figueiredo, et al., 2009).

Outro estudo realizado sobre essa temática demonstrou que $86 \%$ dos alunos do primeiro ano de diversos cursos relataram apreço pela prática de atividades físicas. Porém, esses estudantes afirmavam que não dispunham de tempo livre para a sua realização, evidenciando que a rotina de estudos, de atividades extracurriculares e de extensão, associada a um cronograma de aulas não uniformemente distribuídas ao longo do dia e da semana, podem ser fatores que dificultam o manejo de tempo para a prática de exercícios físicos pelos estudantes (Mielke, et al., 2010).

Desta forma, o objetivo deste estudo foi identificar o perfil de prática de atividades físicas entre estudantes dos sexos feminino e masculino do curso de graduação em medicina da Universidade Federal de Alfenas (UNIFAL-MG).

\section{Metodologia}

Foi realizado um estudo transversal por meio do levantamento de dados primários para avaliação do perfil sociodemográfico e da prática de atividade física entre universitários do curso de medicina. Para tal, foram utilizados instrumentos de coleta de dados padronizados e validados para a população brasileira, os quais foram distribuídos e respondidos por alunos do curso de medicina da UNIFAL-MG, no município de Alfenas, Minas Gerais, Brasil, ingressantes no ano de 2016: 
I) Questionário Sociodemográfico: Foi utilizada uma adaptação da seção A do Composite International Development Interview (CIDI), versão 2.1, para a identificação das seguintes variáveis sociodemográficas: idade, peso, estatura, sexo, estado civil, cor/etnia, atividade profissional e de lazer, apresentar doenças, ter passado por internações, fazer uso de medicamentos, tabagismo, etilismo, e percepção de saúde autorrelatada (Quintana et al., 2007).

II) Questionário Internacional de Atividade Física - Forma Curta (International Physical Activity Questionnaire Short Form - IPAQ-SF): Esse instrumento foi proposto pelo Grupo Internacional para Consenso em Medidas da Atividade Física, constituído sob a chancela da Organização Mundial da Saúde (OMS), com representantes de 25 países, inclusive o Brasil. Trata-se de um instrumento autoadministrado, desenvolvido com a finalidade de estimar o nível de prática habitual de atividade física de populações de diferentes países e contextos socioculturais. A versão curta do IPAQ (IPAQ-SF) é composta por 7 questões abertas e suas informações permitem estimar o tempo despendido, por semana, em diferentes dimensões de atividade física (caminhadas e esforços físicos de intensidades moderada e vigorosa) e de inatividade física (posição sentada) (Matsudo et al., 2001).

No Quadro 1 estão representados os critérios para a classificação em cada nível de atividade física segundo o padrão nacional.

Quadro 1. Classificação do nível de atividade física (IPAQ - forma curta) segundo padrão nacional.

\begin{tabular}{|l|l|}
\hline Classificação & Critérios \\
\hline Muito ativo & $\begin{array}{l}\text { a) Vigorosa: } \geq 5 \text { dias/semana e } \geq 30 \text { minutos por sessão } \\
\text { b) Vigorosa: } \geq 3 \text { dias/semana e } \geq 20 \text { minutos por sessão }+ \text { Moderada } \\
\text { e/ou Caminhada: } \geq 5 \text { dias/semana e } \geq 30 \text { minutos por sessão }\end{array}$ \\
\hline Ativo & $\begin{array}{l}\text { a) Vigorosa: } \geq 3 \text { dias/semana e } \geq 20 \text { minutos por sessão } \\
\text { b) Moderada ou Caminhada: } \geq 5 \text { dias/semana e } \geq 30 \text { minutos por sessão } \\
\text { c) Qualquer atividade somada: } \geq 5 \text { dias/semana e } \geq 150 \text { minutos/semana } \\
\text { (caminhada+moderada+vigorosa) }\end{array}$ \\
\hline Irregularmente ativo A & $\begin{array}{l}\text { Atinge pelo menos um dos critérios da recomendação quanto à } \\
\text { frequência ou à duração (Frequência de } 5 \text { dias/semana ou Duração de } \\
150 \text { minutos/semana) }\end{array}$ \\
\hline Irregularmente ativo B & $\begin{array}{l}\text { Aquele que não atingiu nenhum dos critérios da recomendação quanto à } \\
\text { frequência ou quanto à duração }\end{array}$ \\
\hline Sedentário & $\begin{array}{l}\text { Não realizou nenhuma atividade física por pelo menos 10 minutos } \\
\text { contínuos durante a semana }\end{array}$ \\
\hline
\end{tabular}

Fonte: IPAQ (2007).

Os questionários, utilizados como instrumento de avaliação, foram aplicados em quatro inquéritos, que foram realizados quando os alunos cursavam o início (Q1) e o final (Q2) do segundo período da graduação, e também o início (Q3) e o final (Q4) do terceiro período da graduação.

O estudo em questão foi aprovado pelo Comitê de Ética em Pesquisa da UNIFAL-MG sob parecer número 1.623.096. Todos os participantes do estudo estavam de acordo com a pesquisa e assinaram o Termo de Consentimento Livre e Esclarecido (TCLE) em todas as ocasiões em que foram aplicados os questionários.

O teste estatístico adotado foi o teste Exato de Fisher para comparação das proporções das respostas de cada sexo nos questionários Q1, Q2, Q3 e Q4 e das respostas de cada sexo nos questionários Q1 versus Q2 e Q3 versus Q4. As análises estatísticas foram realizadas no Programa R (R Core Team, 2020) adotando-se o nível de significância de 5\%. 


\section{Resultados e Discussão}

Os dados obtidos por meio do questionário sociodemográfico estão representados na Tabela 1 e, embora os alunos tenham assinado termo de acordo com a pesquisa e as datas de aplicação dos questionários tenham sido previamente agendadas, houve variação no número de questionários respondidos, de forma que os inquéritos Q1, Q2, Q3 e Q4 contaram com a participação de, respectivamente, 22 alunos, 32 alunos, 46 alunos e 45 alunos. Essa diferença no número de dados coletados se deveu principalmente por motivo de faltas, preenchimento inadequado do(s) questionário(s) ou recusa em participar da pesquisa no momento preestabelecido.

A análise das respostas deste questionário demonstrou que os estudantes do Curso de Medicina da Universidade Federal de Alfenas se encontram com idade média em torno dos 21 anos, são solteiros e não mora mais com a família. Esses fatores podem ter influência no perfil de intensidade da prática de atividade física desse grupo. Além disso, vale destacar também a média da distância entre a moradia desses estudantes e o campus da UNIFAL, que variou entre 0,567 e 0,717 km, indicando que possivelmente eles realizam esse trajeto caminhando.

Na Tabela 1 também é possível verificar que o percentual de estudantes com índice de massa corporal (IMC) acima de $24 \mathrm{~kg} / \mathrm{m}^{2}$ oscilou entre 28,13\% e 36,4\%. Embora esse valor de corte do IMC ainda esteja enquadrado como adequado com relação aos valores de referência, vale ressaltar a sua proximidade com o valor definido como sobrepeso (>25 kg/m²) (Kakeshita, 2006). Essa atenção ao IMC se torna um fator importante, pois o desvio padrão dos dados relativos ao IMC assumem valores entre 3,3 e 6,0 .

Tabela 1. Características sociodemográficas e físicas dos estudantes participantes de acordo com o questionário respondido, Alfenas, 2021

\begin{tabular}{|c|c|c|c|c|}
\hline Questionário & Q1 & Q2 & Q3 & Q4 \\
\hline & \multicolumn{4}{|c|}{ Número de participantes } \\
\hline \multirow[t]{2}{*}{$\mathrm{n}$} & 22 & 32 & 46 & 45 \\
\hline & \multicolumn{4}{|c|}{ Tipo de moradia (\%) } \\
\hline República & 63,6 & 78,1 & 84,8 & 76,19 \\
\hline Pensionato & 31,8 & 15,6 & 8,7 & 14,29 \\
\hline \multirow[t]{2}{*}{ Família } & 4,6 & 6,2 & 6,5 & 9,52 \\
\hline & \multicolumn{4}{|c|}{ Distância entre a moradia e a UNIFAL (metros) } \\
\hline \multirow[t]{2}{*}{ Valor médio } & 655,0 & 717,0 & 658,0 & 567,0 \\
\hline & \multicolumn{4}{|c|}{ Estado civil (\%) } \\
\hline Casados & 4,5 & 3,1 & 2,2 & 2,2 \\
\hline Divorciados & 4,6 & 0,0 & 2,2 & 0 \\
\hline \multirow{2}{*}{ Solteiros } & 90,9 & 98,9 & 95,6 & 97,8 \\
\hline & \multicolumn{4}{|c|}{ Índice médio de massa corporal (IMC em kg/m²) } \\
\hline Média \pm desvio padrão & $22,5 \pm 3,6$ & $22,3 \pm 3,6$ & $22,5 \pm 3,3$ & $22,1 \pm 6,0$ \\
\hline \multirow[t]{2}{*}{ Acima de $24 \mathrm{~kg} / \mathrm{m}^{2}(\%)$} & 36,4 & 28,1 & 30,4 & 30,1 \\
\hline & \multicolumn{4}{|c|}{$\operatorname{Sexo}(\%)$} \\
\hline Feminino & 59,1 & 71,9 & 58,7 & 62,2 \\
\hline \multirow[t]{2}{*}{ Masculino } & 40,9 & 28,1 & 41,3 & 37,8 \\
\hline & \multicolumn{4}{|c|}{ Idade em anos } \\
\hline Média \pm desvio padrão & $21,3 \pm 5,1$ & $21,3 \pm 3,4$ & $21,6 \pm 3,8$ & $21,4 \pm 2,5$ \\
\hline
\end{tabular}

Fonte: Dados dos autores. 
Na Tabela 2 estão apresentados os resultados do teste Exato de Fisher (valor-p) obtidos nas comparações entre os sexos dentro de cada um dos questionários e dentro dos sexos em dois momentos diferentes (início e final do semestre), Q1 versus Q2 e Q3 versus Q4.

Tabela 2. Classificação do nível de atividade física (IPAQ-forma curta, padrão nacional) segundo o sexo e questionário e o resultado do teste Exato de Fisher, Alfenas, 2016-2017.

\begin{tabular}{|c|c|c|c|c|c|}
\hline \multirow{3}{*}{ IPAQ } & \multicolumn{4}{|c|}{ Sexo } & \multirow{3}{*}{ Valor-p } \\
\hline & \multicolumn{2}{|c|}{ Feminino } & \multicolumn{2}{|c|}{ Masculino } & \\
\hline & Quant. & $\%$ & Quant. & $\%$ & \\
\hline $\mathrm{Q} 1(\mathrm{n}=22)$ & & & & & 0,9224 \\
\hline Sedentário & 1 & 7,69 & 0 & 0 & \\
\hline Irregularmente ativo B & 2 & 15,39 & 1 & 11,11 & \\
\hline Irregularmente ativo A & 1 & 7,69 & 1 & 11,11 & \\
\hline Ativo & 9 & 69,23 & 6 & 66,67 & \\
\hline Muito ativo & 0 & 0,00 & 1 & 11,11 & \\
\hline Total & 13 & 100,00 & 9 & 100,00 & \\
\hline $\mathrm{Q} 2(\mathrm{n}=32)$ & & & & & 0,6273 \\
\hline Sedentário & 1 & 4,35 & 0 & 0,00 & \\
\hline Irregularmente ativo B & 3 & 13,04 & 3 & 33,34 & \\
\hline Irregularmente ativo A & 3 & 13,04 & 2 & 22,22 & \\
\hline Ativo & 11 & 47,83 & 3 & 33,33 & \\
\hline Muito ativo & 5 & 21,74 & 1 & 11,11 & \\
\hline Total & 23 & 100,00 & 9 & 100,00 & \\
\hline Valor-p & 0,4106 & & 0,5957 & & \\
\hline $\mathrm{Q} 3(\mathrm{n}=46)$ & & & & & 0,8541 \\
\hline Sedentário & 3 & 11,11 & 1 & 5,26 & \\
\hline Irregularmente ativo B & 3 & 11,11 & 1 & 5,26 & \\
\hline Irregularmente ativo A & 3 & 11,11 & 3 & 15,79 & \\
\hline Ativo & 15 & 55,56 & 11 & 57,90 & \\
\hline Muito ativo & 3 & 11,11 & 3 & 15,79 & \\
\hline Total & 27 & 100,00 & 19 & 100,00 & \\
\hline $\mathrm{Q} 4(\mathrm{n}=45)$ & & & & & 0,4471 \\
\hline Sedentário & 2 & 7,14 & 3 & 17,65 & \\
\hline Irregularmente ativo B & 3 & 10,72 & 3 & 17,65 & \\
\hline Irregularmente ativo A & 8 & 28,57 & 2 & 11,76 & \\
\hline Ativo & 13 & 46,43 & 9 & 52,94 & \\
\hline Muito ativo & 2 & 7,14 & 0 & 0,00 & \\
\hline Total & 28 & & 17 & 100,00 & \\
\hline Valor-p & 0,5951 & & 0,3099 & & \\
\hline
\end{tabular}

Nota: valor-p na vertical corresponde à análise entre sexos, na horizontal intra sexo

Fonte: Dados dos autores.

Os dados apontaram que em Q1 há uma proporção considerável de mulheres no nível de atividade "ativo", 69,23\% e que o segundo maior valor equivale à proporção de 15,39\% da categoria "Irregularmente ativo B". A proporção masculina no nível "ativo" é igual a $66,67 \%$ e três das demais categorias as proporções são iguais $11,11 \%$ e "sedentário", $0,00 \%$. Comparando-se os dois sexos quanto às proporções de respostas das categorias IPAQ padrão nacional, observou-se não existir diferenças estatísticas ( $\mathrm{p}=0,9224)$ entre ambos os sexos. Em Q2 notou-se que a proporção de mulheres no nível de atividade "ativo" manteve um valor numericamente maior do que nas demais categorias, 47,83\%. Neste momento, houve valores proporcionais iguais numericamente das categorias IPAQ padrão nacional para os homens em duas delas: "Irregularmente ativo B" e "ativo", 33,34\%. Estatisticamente, os dois grupos, feminino e masculino, não se diferiram estatisticamente 
( $\mathrm{p}=0,6273$ ). Pelos questionários Q1 e Q2 no grupo feminino observou-se não haver, estatisticamente, diferenças ( $\mathrm{p}=0$,4106) entre as proporções de IPAQ padrão nacional. Resultado semelhante pôde ser observado no grupo masculino ( $\mathrm{p}=0,5957$ ) nestes questionários. No questionário Q3 observou-se proporções semelhantes entre a categoria "ativo" entre os dois sexos, valores iguais a 55,56\% e 57,90\%, para o grupo feminino e masculino, respectivamente. A análise estatística não acusou diferenças significativas $(\mathrm{p}=0,8541)$ entre os grupos nas diferentes categorias de IPAQ padrão nacional. O último questionário aplicado, Q4, também não se notou diferenças estatisticamente significativas $(\mathrm{p}=0,4471)$ entre os dois sexos nas diferentes categorias de IPAQ padrão nacional. Dentro dos sexos, observou-se que os grupos femininos comparados em Q3 e Q4 não se diferiram estatisticamente $(p=0,5951)$ e, semelhantemente, os grupos masculinos $(p=0,3099)$. Os resultados estatísticos permitem concluir que, embora as distribuições das proporções de cada sexo dentro de cada questionário sejam numericamente diferentes, estas proporções comparadas com outro grupo indicam haver semelhanças estatísticas entre os grupos testados estatisticamente. Isto significa haver padrões comportamentais semelhantes em relação à atividade física, ou seja, ao mesmo tempo que uma categoria de IPAQ apresenta proporção alta em grupo de sexo, ela também é alta em outro grupo, e valores proporcionais baixos em um grupo são também em outro grupo.

Bastos Neto, et al. (2020), encontraram resultados numericamente semelhantes aos resultados apresentados na Tabela 2, quanto aos estudantes de medicina classificados como "ativos", com percentual de cerca de 53\%. Ainda nesse estudo foi demonstrado também que $26 \%$ foram classificados como "irregularmente ativos" e outros $16 \%$ foram classificados como “sedentários". Em contrapartida, o estudo de Pires, et al. (2013), realizado com estudantes de enfermagem, apresentou resultados proporcionalmente opostos aos observados na Tabela 2, ou seja, a maioria dos estudantes foi classificada como "sedentário". Castro Júnior et al. (2012) também chegaram a resultados semelhantes aos citados anteriormente, pois demonstraram que a maioria dos estudantes de Medicina foi classificada como "sedentário" ou "insuficientemente ativo", representada por uma parcela de 73,1\%. Já em uma comparação entre o sexo feminino e masculino, houve uma prevalência do sedentarismo entre o sexo feminino, representado por uma parcela de 63,1\% das mulheres, contra $45,1 \%$ dos homens, resultado que indica uma similaridade parcial com presente estudo, visto que em Q4 as mulheres apresentaram valores proporcionais de sedentarismo menores do que os apresentados pelos homens. No estudo também foi abordado por como principal motivo para não praticar atividades físicas a falta de tempo, relatado por $74,6 \%$ dos estudantes, devido à elevada carga horária dedicada ao curso.

Já segundo Serinolli et al. (2015), foi possível identificar que dentre os alunos do primeiro ao sexto anos, 40,78\% dos alunos não realizam atividades físicas por pelo menos trinta minutos, em contrapartida que 28,64\% realizam até duas vezes por semana e 30,62\% realizam por três ou mais vezes por semana. Além disso, constatou-se que os estudantes de medicina que praticam exercícios físicos e participam de competições esportivas apresentaram melhor domínio físico, psicológico, relação social e ambiental quando comparado com o grupo que não realiza nenhum tipo de atividade física. Essa relação positiva entre a prática de atividade física e a melhora na qualidade de vida também foi encontrada no estudo de Peleias (2018), que da mesma forma associou os maiores índices de atividade física entre homens em detrimento das mulheres.

Mendes-Netto, et al., em estudo realizado em 2012, demonstraram resultados semelhantes ao presente estudo quanto ao fato de que o grupo de homens se apresentaram mais ativos que o de mulheres estudantes da área da saúde. Em relação ao nível "muito ativo", foram encontrados $24,5 \%$ dos homens e $16,1 \%$ das mulheres na amostra sob análise; o nível "ativo" foi representado por 48,2\% dos homens e 42,1\% das mulheres; os padrões "insuficientemente ativo" tipos A e B foram encontrados em $23,6 \%$ dos homens e $28,5 \%$ das mulheres; e, por fim, o nível "sedentário" era de 3,6\% dos homens e 13,2\% das mulheres.

Em relação ao tempo gasto por dia na prática de exercícios físicos, nota-se na Tabela 3 um valor numérico maior da média tempo dedicado à caminhada no momento de aplicação dos questionários Q2 e Q3, enquanto em Q4 esse tempo diminui 
(24,44 minutos), mas se mantém maior que no início da pesquisa (22,73 minutos). O tempo dedicado a atividades moderadas seguiu valores similares, com um tempo médio de 44,67 minutos quando foi aplicado o inquérito Q4. Já para as atividades vigorosas, o tempo gasto sofreu uma diminuição numérica na média de 2,38 minutos ao decorrer da pesquisa.

Tabela 3. Média de tempo gasto na prática dos diferentes tipos de atividade física.

\begin{tabular}{cccc}
\hline $\begin{array}{c}\text { Momento do } \\
\text { inquérito }\end{array}$ & Caminhada & Atividade Física (min/dia) \\
\hline Q1 & 22,73 & 36,14 & Atividade Vigorosa \\
Q2 & 38,44 & 63,44 & 46,82 \\
Q3 & 32,39 & 52,72 & 38,44 \\
Q4 & 24,44 & 44,67 & 45 \\
\hline
\end{tabular}

Fonte: Elaborado pelos autores com base nos dados obtidos nos questionários.

Os resultados obtidos possuem resultados semelhantes aos achados de uma pesquisa realizada por Figueiredo et al (2009) para as atividades vigorosas e caminhada, em que a primeira sofreu redução do tempo despedido com a sua prática e a segunda sofreu aumento seguido de redução do tempo dedicado à sua prática. Já o tempo para as atividades moderadas sofreu queda pelos dados obtidos no presente trabalho, conforme discutido anteriormente, o que diverge dos resultados apresentados por Figueiredo et al (2009).

\section{Considerações Finais}

Ainda que se considere as consequências dos diferentes desafios e exigências que o curso de graduação em medicina oferece, bem como condições pessoais de cada aluno, os resultados não apontaram diferenças estatísticas das classificações entre homens e mulheres, tampouco entre cada período dentro do mesmo sexo. No entanto, dada a importância em abordar esse tema, observou-se uma diminuição da proporção de alunos no nível de atividade "ativo" em contraste com o aumento no nível "Irregularmente ativo A" aos finais dos períodos letivos, o que pode ter como justificativa o acúmulo e sobreposição das atividades curriculares, característicos nessa época.

Logo, conclui-se que, em face aos diferentes desafios encontrados ao ingressar na universidade, como a mudança da rotina, distância de casa e o enfrentamento de exigências cada vez maiores com o avanço do curso, o acadêmico de medicina pode encontrar dificuldades em manter o mesmo padrão de atividade física. Conforme discutido, esse fato possui influências diretas sobre sua saúde física e mental, o que confirma a importância desse estudo e a necessidade de uma maior atenção ao tema.

Vale evidenciar também a importância de estudos que abordem melhor a prática de atividades físicas entre adultos e universitários, devido à dificuldade em encontrar, na literatura, trabalhos que abordem esse tema. Ademais, é válido ressaltar a necessidade de estudos longitudinais no tema, pois foi abordado um grupo relativamente pequeno e também com uma amostra temporalmente limitada.

\section{Referências}

Alves, C. R., das Neves, W., de Almeida, N. R., Eichelberger, E. J., Jannig, P. R., Voltarelli, V. A., \& Hagen, L. (2020). Exercise training reverses cancerinduced oxidative stress and decrease in muscle COPS2/TRIP15/ALIEN. Molecular metabolism, 39, 101012. doi.org/10.1016/j.molmet.2020.101012

Barros-Neto, T. L. (2003). Sedentarismo. http://www.emedix.com.br/doe/mes001_1f_sedentarismo.php 
Bastos Neto, P. \& Campos, G. A. L. (2020). Avaliação da influência acadêmica sobre a prática de atividades físicas nos estudantes de medicina em uma faculdade do noroeste do estado de São Paulo. Revista Corpus Hippocraticum, 1(1).

Bonfim, V. S. G.; Bianchi, L. R. O.; Mello, S. T.; \& Sant'Ana, D. M. G. (2019). A Importância do Exercício Físico para Neuroplasticidade e Aprendizado. Arquivos do Mudi, 23(3), 189-200.

Castro Júnior, E. F. D., Barreto, L. A., Oliveira, J. A. A., Almeida, P. C., \& Leite, J. A. D. (2012). Evaluación del nivel de actividad física y factores asociados en estudiantes de medicina de Fortaleza-CE. Revista Brasileira de Ciências do Esporte, 34(4), 955-967. doi.org/10.1590/S0101-32892012000400011.

Figueiredo, E. T. de, Morais, A. M. de, \& Costa, A. M. D. D. (2009). Influência da rotina acadêmica na prática de atividade física em graduandos de Medicina. Revista Brasileira Clínica Médica, 7, 174-176.

IPAQ (2007). Classificação do Nível de Atividade Física IPAQ. https://edisciplinas.usp.br/pluginfile.php/3343547/mod_resource/content/1/IPAQ.pdf

Kakeshita, I. S., \& Almeida, S. D. S. (2006). Relação entre índice de massa corporal e a percepção da autoimagem em universitários. Revista de Saúde Pública, 40(3), 497-504. doi.org/10.1590/S0034-89102006000300019.

Matsudo, S. M. (2001). AT, Matsudo VK, Andrade D, Andrade E, Oliveira LC, et al. Questionário Internacional de Atividade Física (IPAQ): estudo de validade e reprodutibilidade no Brasil. Revista Atividade Física \& Saúde, 6(2), 5-18.

Mendes-Netto, R. S., da Silva, C. S., Costa, D., \& Raposo, O. F. F. (2012). Nível de atividade física e qualidade de vida de estudantes universitários da área de saúde. Revista de Atenção à Saúde, 10(34), 47-55. doi.org/10.13037/rbcs.vol10n34.1802

Mielke, G. I., Ramis, T. R., Habeyche, E. C., Oliz, M. M., Tessmer, M. G. S., Azevedo, M. R., \& Hallal, P. C. (2010). Atividade física e fatores associados em universitários do primeiro ano da Universidade Federal de Pelotas. Revista Brasileira de Atividade Física \& Saúde, 15(1), 57-64.

doi.org/10.12820/rbafs.v.15n1p57-64

Pate, R. R., O'neill, J. R., \& Lobelo, F. (2008). The evolving definition of" sedentary". Exercise and sport sciences reviews, 36(4), 173-178. doi.org/10.1097/J E S.0b013e3181877d1a

Peleias, M. D. D. A. (2018). A atividade física e a qualidade vida do estudante de medicina no Brasil (Tese de doutorado). Universidade de São Paulo, São Paulo.

Pires, C. G. D. S., Mussi, F. C., Cerqueira, B. B. D., Pitanga, F. J. G., \& Silva, D. O. D. (2013). Prática de atividade física entre estudantes de graduação em enfermagem. Acta Paulista de Enfermagem, 26(5), 436-443. doi.org/10.1590/S0103-21002013000500006.

Pitanga, F. G. (2008). Epidemiologia, atividade física e saúde. Revista Brasileira de Ciência e Movimento, 10(3), 49-54.

Quintana, M. I., Gastal, F. L., Jorge, M. R., Miranda, C. T., \& Andreoli, S. B. (2007). Validity and limitations of the Brazilian version of the Composite International Diagnostic Interview (CIDI 2.1). Brazilian Journal of Psychiatry, 29(1), 18-22. 10.1590/S1516-44462006005000024

R Core Team (2020). R: A language and environment for statistical computing. R Foundation for Statistical Computing, Vienna, Austria. URL https://www.Rproject.org/.

Reis, R. S., Petroski, E. L., \& Lopes, A. D. S. (2000). Medidas da atividade física: revisão de métodos. Rev Bras Cineantropom Desempenho Hum, 2(1), 8996.

Ropke, L. M., Souza, A. G., de Magalhães Bertoz, A. P., Adriazola, M. M., Ortolan, E. V. P., Martins, R. H., \& Weber, S. A. T. (2018). Efeito da atividade física na qualidade do sono e qualidade de vida: revisão sistematizada. Archives of Health Investigation, 6(12), 561-568. 10.21270/archi.v6i12.2258.

Serinolli, M. I., \& El-Mafarjeh, E. (2015). Impacto da prática de atividade física na qualidade de vida dos acadêmicos de Medicina da Universidade Nove de Julho (Uninove). ConScientiae Saúde, 14(4), 627-633. doi.org/10.5585/conssaude.v14n4.5958

Silva, R. A. D., Horta, B. L., Pontes, L. M., Faria, A. D., Souza, L. D. D. M., Cruzeiro, A. L. S., \& Pinheiro, R. T. (2007). Bem-estar psicológico e adolescência: fatores associados. Cadernos de Saúde Pública, 23, 1113-1118. doi.org/10.1590/S0102-311X2007000500013

Veigas, J., \& Gonçalves, M. (17/07/2009). A influência do exercício físico na ansiedade, depressão e stress. Portal dos Psicólogos, 1 -19. https://www.psicologia.pt/artigos/textos/A0485.pdf 